\title{
Acute Renal Infection in Adult, Part 1: An Overview of What the Radiologist Needs to Know
}

\author{
Suman Hazarika ${ }^{1}$ Rochita Venkataramanan² ${ }^{2}$ Tonmoy Das ${ }^{3} \quad$ Sukanya Deuri ${ }^{1} \quad$ Shalini Lohchab ${ }^{1}$ \\ Tamsir Rongpipi ${ }^{1}$ Asish Agarwala ${ }^{1}$ Akash Venkataramanan ${ }^{4}$
}

${ }^{1}$ Department of Radiology, Apollo Hospitals Guwahati, Guwahati, Assam, India

2Department of Radiology, Apollo Hospitals Chennai, Chennai, Tamil Nadu, India

${ }^{3}$ Department of Nephrology, Apollo Hospitals Guwahati, Guwahati, Assam, India

${ }^{4}$ Medical school, Madras Medical College Chennai, Chennai,

Tamil Nadu, India

J Gastrointestinal Abdominal Radiol ISGAR:2020;3:126-136
Address for correspondence Rochita Venkataramanan, MD, DNB, DMRD, No. 34 Srinivasa Murthy Avenue, Off LB Road, Adyar, Chennai 600020, Tamil Nadu, India (e-mail: rochitav@gmail.com).

\begin{abstract}
Acute renal infection or acute pyelonephritis (AP) denotes the process of inflammation of the renal parenchyma and its collecting system and the urothelium following infection. Uncomplicated AP commonly affects otherwise healthy, young women without structural or functional urinary tract abnormalities and without relevant comorbidities. More severe and complicated AP occurs in patients with a structurally or functionally abnormal genitourinary tract, or in persons with a predisposing medical condition like immune compromised state and diabetes. Complicated AP is characterized by a broader spectrum of clinical presentations, a wider variety of infecting organisms, and a greater risk of progression to a complication, such as intrarenal or perinephric abscess or emphysematous pyelonephritis and has the capacity to damage the organ and at times maybe life threatening. Role of imaging in renal infection is secondary, and in most situations, imaging is done to confirm the clinical diagnosis, map progression of disease in immune-compromised group of patients, or to evaluate for poten-

Keywords

- acute pyelonephritis

- papillary necrosis

- pyonephrosis

- renal abscess tial complications and therapeutic interventions. This article attempts to discuss the pathophysiology of AP from the standpoint of medical imaging and also brings out illustrative examples of various manifestations of AP and its complications. It provides imaging insight into various stages of inflammation, development of complication, and a roadmap for understanding AP through cross-sectional imaging.
\end{abstract}

\section{Introduction}

Acute renal infection or acute pyelonephritis (AP) denotes the process of inflammation of the renal parenchyma and its collecting system and the urothelium following infection. Uncomplicated AP commonly affects otherwise healthy, young women without structural or functional urinary tract abnormalities and without relevant comorbidities. More severe and complicated AP occurs in patients with a structurally or functionally abnormal genitourinary tract, or in persons with a predisposing medical condition like immune compromised state and diabetes. Complicated AP is characterized by a broader spectrum of clinical presentations, a wider variety of infecting organisms, and a greater risk of progression to a complication, such as intrarenal or perinephric abscess or emphysematous pyelonephritis ${ }^{1}$ and has the capacity to damage the organ and at times maybe life threatening. As histological specimens are difficult to obtain, clinical correlation with stages of inflammation and developing complication is nearly impossible. In day-to-day 
clinical practice, the primary goal of imaging is to provide information about the nature and extent of the disease and to identify significant complications, for example, gas-forming infection, abscess, and urinary obstruction. ${ }^{2}$

Ultrasonography (USG) is the most widely used screening tool, although it is limited in its ability to map disease progression and suffers from operator bias. Currently, computed tomography (CT) with or without CT urography and magnetic resonance imaging (MRI) find wide acceptance for diagnosis of AP and its potential complications. This article attempts to discuss the pathophysiology of AP from the standpoint of medical imaging and also brings out illustrative examples of various manifestations of AP and its complications. It provides imaging insight into various stages of inflammation, development of complication, and a roadmap for understanding AP through cross-sectional imaging.

\section{Acute Pyelonephritis: Terminological Conundrum}

Numerous verbal expressions have added to the confusion about the terminology used to describe the extent and severity of AP. Popular terminology used by radiologists was based solely on imaging appearance without histological confirmation. The advanced generalized form of AP in diabetic patients, usually with a nonfunctioning kidney on excretory urography, was originally described as acute bacterial pyelonephritis. ${ }^{3}$ The localized form of AP resulting in a mass-like appearance in imaging studies was given numerous names including acute focal pyelonephritis, acute focal bacterial nephritis, and acute lobar nephronia ${ }^{4,5}$ (-Fig. 1).

As cross-sectional modalities have evolved, subtle abnormal nephrographic findings were picked up variably termed as cellulitis, carbuncle, and renal phlegmon. In 1984, Talner et $\mathrm{al}^{6}$ proposed that all renal parenchymal abnormalities without formation of abscess attributable to acute infection maybe called AP. It was proposed that degree of involvement should be described by one or more of the following modifiers: (1) unilateral or bilateral, (2) focal or diffuse, (3) focal swelling or no focal swelling, and (4) renal enlargement or none.

\section{Acute Pyelonephritis: Must Know Clinical Issues}

Clinically AP is suggested by flank pain, nausea, and vomiting, fever $\left(>38^{\circ} \mathrm{C}\right)$, or costovertebral angle tenderness, and it can occur in the absence of symptoms of cystitis.?

AP in pregnant women may not only develop into renal and respiratory insufficiency, but also there is added risk of frequent preterm labor. ${ }^{8}$ It is of great importance to diagnose AP confidently and pick up complications early to provide effective treatment. Most men with febrile urinary tract infection (UTI) develop concomitant prostatitis. ${ }^{9}$ Prostate infections are notoriously difficult to cure and need long-term antibiotics. A diagnosis of AP in a man should remind us to image the prostate, preferably by transrectal USG or a diffusion-weighted MRI (DWI). Diabetic patients with AP are at risk of developing

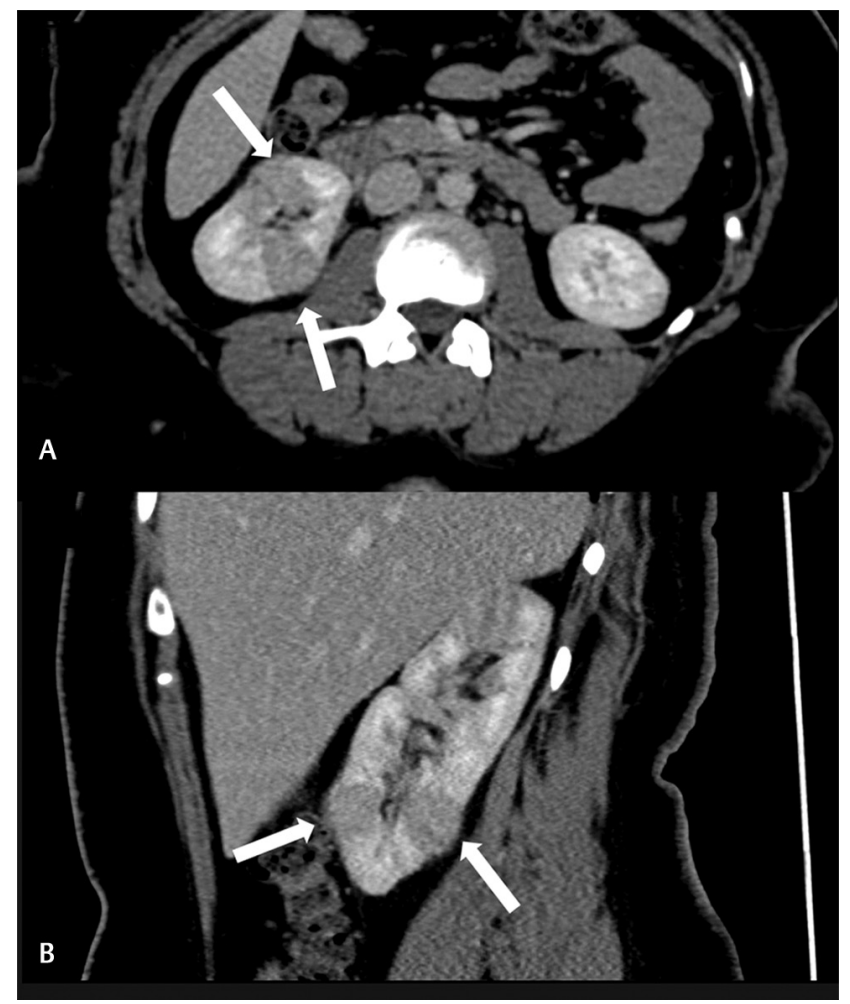

Fig. 1 (A) Axial and sagittal (B) CT sections in the nephrographic phase of a 52-year-old diabetic man show round mass like areas of reduced enhancement representing lobar nephronia (arrows).

hypo- and hyperglycemia, hyperosmolar dehydration, or ketoacidosis ${ }^{10}$ and also infection by gas-forming organisms with a high mortality (emphysematous pyelonephritis). Emphysematous pyelonephritis is characterized histologically by acute pyogenic infiltration with microabscesses and the development of acute renal failure. ${ }^{11}$ Laboratory findings of AP include urinary pus cells in excess, granular or leukocytic casts, bacteriuria, and a positive urine culture. Blood tests may show leukocytosis with a neutrophilic shift, elevated erythrocyte sedimentation rate, elevated C-reactive protein levels, and occasionally positive blood cultures that grow the same organism as cultured from the urine.

\section{Acute Pyelonephritis: Pathology and Pathogenesis}

The heralding event is inoculation of the urinary bladder with an infectious organism, which then migrates up the ureter to the central collecting system. Vesicoureteral reflux (VUR) is the retrograde flow of urine from the bladder into the ureter and toward the kidney secondary to a dysfunctional vesicoureteric junction (VUJ). The VUJ normally acts as a one-way valve, allowing urine flow from the ureter into the bladder and closing during micturition, preventing back flow. Shorter intramural submucosal segment of distal ureter increases the likelihood of VUR. The ratio of ureteric submucosal tunnel length and ureteric diameter critically determines the valve action. In healthy individual, the ratio is 5:1, while in most patients with primary VUR, it is $\sim 1.4: 1$. The ascent of infection from bladder to kidney occurs even in 
the absence of VUR, owing to special virulence properties of the bacteria such as the adhesin P fimbriae and endotoxins. The bacterial endotoxins are believed to inhibit ureteral peristalsis by inhibiting the adrenergic nerves that supply the smooth muscle of the ureter. The ureter as a result lays flaccid creating a functional obstruction and urinary stasis. The ureteric obstruction compromises the forward flow of urine, which is a normal protective mechanism against upper UTI. Infection originates from the lower urinary tract and ascends via subepithelial lymphatic channels or directly via the ureter. Escherichia coli (E. coli) is the most common pathogen. In elderly patients with a history of instrumentation, however, Proteus mirabilis is a frequent offender. E. coli has the ability to attach to urothelial surfaces via pili or fimbriae. Once the kidneys are infected there is either global renal enlargement or sometimes there is patchy areas of inflammation in different stages. Typically, the pelvicalyceal system is involved first (pyelitis). The renal interstitial inflammation starts from the medullary region toward the cortex. Hematogenous infection occurs less commonly, where the renal cortex is involved first.

Complications of AP include abscess formation and spread of infection to perirenal space and beyond.

\section{Imaging of Acute Pyelonephritis and Its Complications}

\section{When Do You Image Acute Renal Infection?}

Mostly UTIs are treated without imaging as the diagnosis is straightforward. In confounding situations where significant clinical remissions are not seen following antibiotic therapy of 3 days, imaging is called for. ${ }^{12,13}$ In the situation when a definite diagnosis of AP is not established or when patients present with recurrent episodes of infection, renal imaging is indicated because there is more likelihood of stones, obstruction, abscess, or a congenital anomaly.

Imaging is also requested in a select group of patients where risk of complications runs high.

Patients with poorly controlled diabetes mellitus, acquired immunodeficiency syndrome, renal transplantation, or other immunocompromised disease states are considered at risk of developing a complicated UTI, including development of renal or perinephric abscess. Therefore, imaging may be required when such patients present initially. ${ }^{14,15}$ Special mention must be made about the case of diabetes. Apart from risk of abscess formation and extrarenal infection spread, diabetics warrant early imaging in UTI. Up to 50\% may not have the typical flank tenderness that helps to differentiate AP from a lower UTI. Absence of flank pain in diabetics might be a critical confounding factor and vigil against missing the narrow window of opportunity to initiate early treatment must be kept. ${ }^{12,16}$

\section{Role of Ultrasonography in Acute Pyelonephritis}

USG is one of the first imaging investigations to be ordered because it is easily available and gives a quick look helping to rule out any predisposing factors like obstruction as well as intra or perirenal collections. However, signs of early AP may not be visible on USG and mild swelling as well as loss of corticomedullary differentiation present in such cases is subjective and may be missed.

A large study from South India on role of emergency ultrasound screening (EUS) in the evaluation of 1,218 patients with clinically suspected AP found that nearly $49 \%$ had a normal EUS, while $51 \%$ had at least one major or minor abnormality. The frequency of hydroureteronephrosis, renal calculi emphysematous changes, and renal abscess was 19.1, 8.9, 2.1, and $1.9 \%$, respectively. Only $5.9 \%$ patients required emergency percutaneous nephrostomy or drainage of an abscess. They concluded that a large proportion of AP patients has only normal or minor abnormalities and do not need additional screening and intervention. ${ }^{17}$

\section{CT versus MRI-How Do You Image Acute Pyelonephritis?}

The American College of Radiology appropriateness criteria for AP (revised 2018) state that CT abdomen and pelvis with IV contrast or $\mathrm{CT}$ abdomen and pelvis without and with intravenous (IV) contrast are usually appropriate for imaging complicated patients in the setting of AP. ${ }^{18}$ It also considers imaging investigation as "usually not appropriate" in uncomplicated cases. CT is a sensitive tool for AP and its sequelae, and it also provides a global assessment of the abdomen for coincidental pathology. Renal obstruction by urolithiasis is well picked up by CT apart from its ability to provide superior anatomic detail for underlying congenital, as well as acquired renal abnormalities. ${ }^{19}$

Ability of contrast-enhanced CT to predict parenchymal changes, change in renal perfusion, and function are useful predictors of clinical outcome. Studies comparing CT with USG conclude that additional benefit of CT lies in its ability to detect parenchymal abnormalities in patients with AP that are generally missed by USG ${ }^{19,20} \mathrm{CT}$ scores over MRI in its ability to detect calculi and gas in pyelonephritis. Soulen et $\mathrm{al}^{13}$ emphasized validity of waiting for 72 hours prior to obtaining CT in patients with AP. In their series, $95 \%$ of patients with uncomplicated AP became afebrile within 48 hours of appropriate antibiotic therapy, and nearly $100 \%$ did so within 72 hours. In situations where use of iodinated contrast material must be avoided, MRI is fast emerging as an alternative to $\mathrm{CT}$ in imaging $\mathrm{AP}$.

\section{Role of DWI}

In biological tissues that are highly cellular (such as tumors), the higher density of cell membranes restricts the diffusion of water protons. This restriction to diffusion manifests as high signal intensity on DWI and corresponding lower apparent diffusion coefficient (ADC). Use of ADC can serve as a noninvasive sensitive marker for renal fibrosis as an apparent decrease in ADC is associated with increased number of fibroblasts. Currently, it has been first author's institutional practice to obtain DWI in situations where use of IV contrast is risky in diabetics with borderline glomerular filtration rate, pregnant women suspected of UTI, children with UTI showing prolonged fever and not responding to treatment. Comparison of DWI versus contrast-enhanced MRI was done for pediatric 
population with equivalent outcome for both techniques. ${ }^{21}$ Rathod et al did a prospective study with DWI in a series of cases and found a higher sensitivity of DWI (95\%) compared with noncontrast CT $(67 \%)$ and contrast-enhanced CT (88\%) in the diagnosis of AP. Areas of AP show significantly lower $A D C$ values than normal renal cortical parenchyma. Renal abscesses show significantly lower ADC values than areas of AP. ${ }^{22}$ Similar observations of high sensitivity, specificity, and accuracy (each at 95\%) with DWI in uncomplicated AP were reported by other studies too. ${ }^{23}$ Another exciting application of DWI is that of differentiation of pyonephrosis from hydronephrosis. Low ADC values of pyonephrosis debris were found particularly helpful for pregnant patients in the second and third trimesters who may have physiological hydronephrosis due to compression by the gravid uterus by Chan et al. ${ }^{24}$ Prediction of renal function by DWI is found to be a reproducible technique by Thoeny et al. ${ }^{25}$

\section{CT Protocol for Acute Pyelonephritis}

Craig et $\mathrm{al}^{19}$ and Stunell et $\mathrm{a}^{26}$ have studied pyelonephritis by only precontrast and nephrographic phase CT in cases where obstruction is not suspected. Taniguchi et $\mathrm{al}^{27}$ reported that scans using only the nephrographic phase had high accuracy with triphasic scans (which also included precontrast and excretory phases) for the diagnosis of AP and urolithiasis. This study reported an accuracy of nephrographic phase only CT of 90 to $92 \%$ in the diagnosis of AP and CT of 96 to $99 \%$ in the diagnosis of urolithiasis. Currently in our institutions, we justify use of unenhanced CT for detecting calculi, gas-forming infections, and hemorrhage. First and second authors institutional protocol includes a nephrographic phase at 60 to 90 seconds and excretory phase at 5 to 10 minutes post IV contrast injection studies to completely evaluate patients with renal inflammatory disease and also to define changes in the renal excretion of the contrast material that occur as a result of the inflammation. Papillary necrosis that is not uncommon is also better evaluated in the excretory phase. Our current experience also dictates that certain findings like streaky nephrogram, delayed parenchymal enhancement, and urothelial thickening are well picked up in excretory phase images.

Second author's institutional protocol additionally includes a corticomedullary phase at 25 to 30 seconds postIV contrast injection to cover any vascular complications like pseudoaneurysms, preoperative arterial mapping if any surgical intervention is required and assessing incidentally detected cysts and masses ( - Fig. 2 ).

\section{MRI Protocol for Acute Pyelonephritis}

At First author's institution, DWI is used with multiple b values $(-50,600,1,000)$. The protocol includes fat-saturated T2 in axial and T2 coronal, axial, and coronal LAVA (which gives in and opposed phase images, Water LAVA T1, Fat LAVA T1) and in most cases axial and coronal FS T1 following IV Gadolinium.

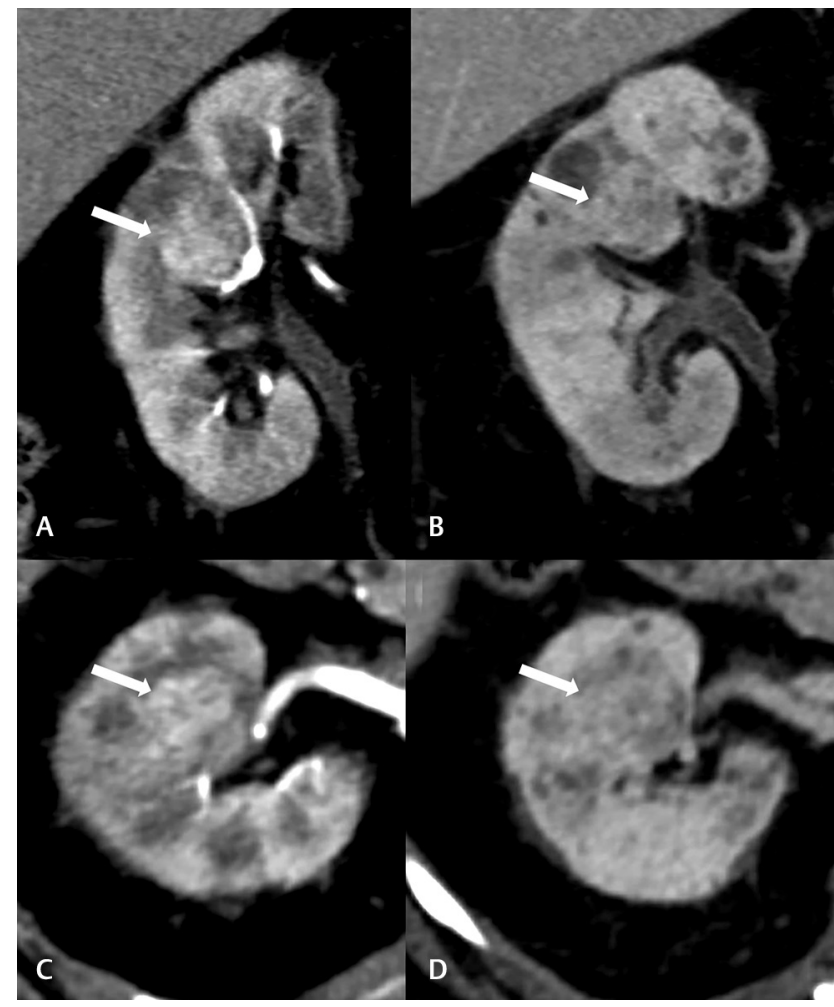

Fig. 2 A 68-year-old nondiabetic man with AP. Panels A (coronal) and B (axial) in the corticomedullary or arterial phase show an incidentally detected small renal cell carcinoma (arrows) that was not picked up by ultrasonography possibly because of adjacent architectural changes and heterogeneity caused by the AP. Panels $\mathbf{C}$ (coronal) and D (axial) in the nephrographic phase show that the mass is isodense to the parenchyma (arrows) and difficult to identify highlighting the importance of the corticomedullary phase.

\section{Imaging Findings of Acute Pyelonephritis: Illustrative Cases}

In first author's institutional experience of over two decades and retrospective analysis of 100 cases of AP, the commonest imaging findings were global renal swelling (88\%), perirenal fascial thickening (76\%), perinephric fat stranding (56\%), streaky nephrogram (44\%), frank abscess (44\%), microabscess (32\%), gas in renal collecting system or parenchyma suggestive of emphysematous infection (16\%), and abdominal wall involvement (8\%).

\section{Streaky Nephrogram}

The common CT finding of striated or streaky nephrogram (-Fig. 3) is seen as linear bands of alternating hyper- and hypoattenuation orientated parallel to the axis of the tubules and collecting ducts. After administration of contrast material, this pattern of differential enhancement reflects the underlying pathophysiology of tubular obstruction caused by inflammatory debris within the lumen, interstitial edema, and vasospasm. All three of these pathophysiologic disturbances tend to decrease the flow of contrast agent through the renal tubule, which also helps explain the pattern of delayed and persistent enhancement seen 3 to 6 hours after administration of contrast material. The sites that originally 


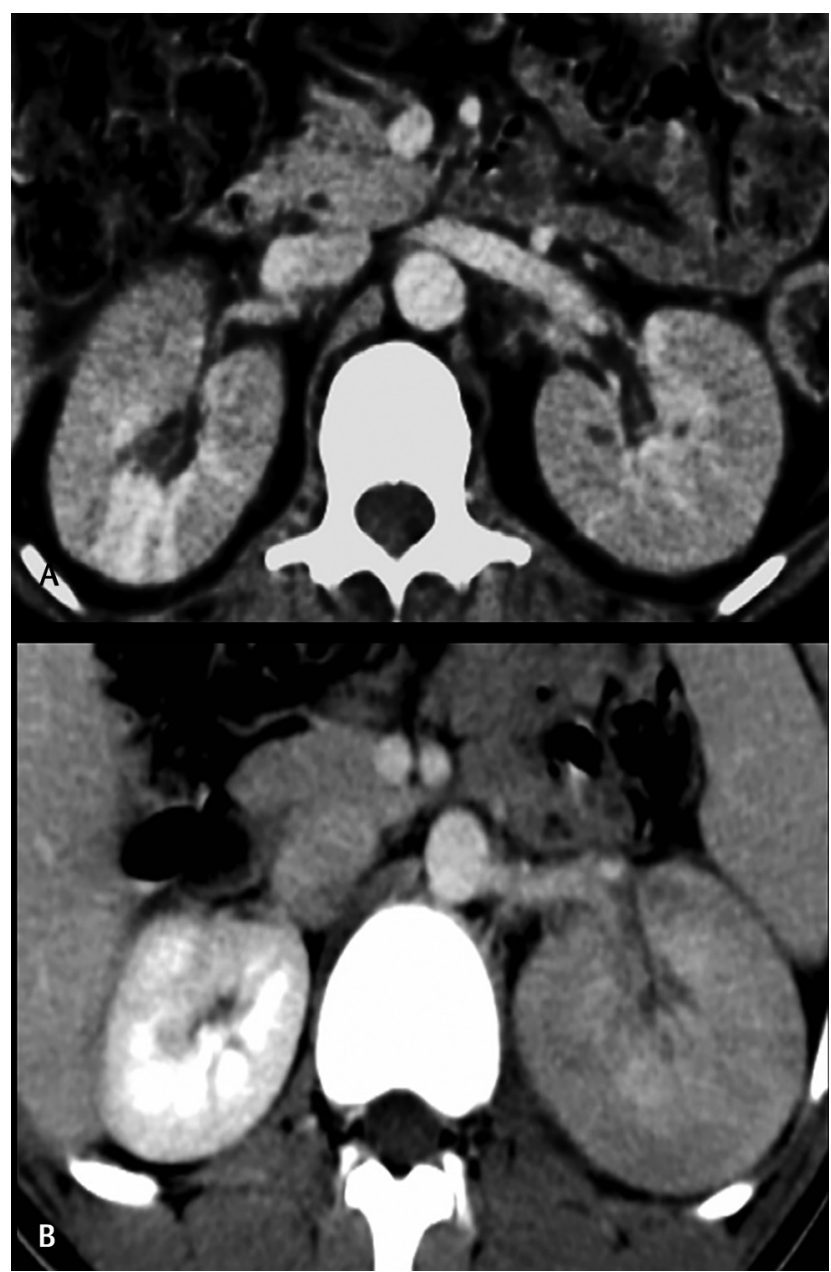

Fig. 3 A young woman presenting with fever with chill and rigor, dysuria, and increased frequency. Nephrographic phase axial CT shows alternating bands of hypo- and hyperattenuation in both the kidneys in panel (A) and patchy enhancement with alternate hypoand hyperattenuation areas in left kidney (B).

demonstrated reduced attenuation during the nephrographic phase transmute from hypoattenuation to hyperattenuation wedge-shaped defects because of the prolonged accumulation of contrast agent that slowly transits the compromised tubules. The learning point here is that though a streaky nephrogram is taught to be associated with AP, it is also common to be seen in case of renal vein thrombosis and acute renal or ureteric obstruction. Visualization of a thrombus in the renal vein and an anatomical obstruction or calculi in the ureter are not difficult to pick up on CT. In our experience, streaky nephrogram or hypoperfused areas may not be well seen in initial nephrographic phase in some situations, but may be better depicted in narrow window settings in the excretory phase images ( - Figs. $\mathbf{4 A - C}, \mathbf{5 A}$ and $B$ ).

\section{Renal Swelling}

The finding of renal swelling is best assessed by experience rather than measurement ( - Fig. 6). Although renal swelling is a common feature, not all cases of AP present with swollen kidney. Perinephric fat stranding and perirenal fascial thickening are also found by the authors to be contributory but nonspecific findings.

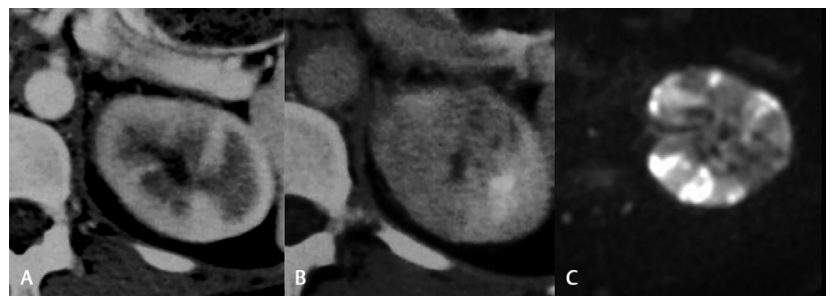

Fig. 4 Near normal nephrographic phase axial CT image (A). In narrow window setting the heterogenous parenchymal enhancement is depicted in a delayed phase axial CT image (B). Complimentary diffusion-weighted imaging was done that reveals a dramatic appearance of alternate bright and hypointense areas suggesting restricted diffusion in renal parenchyma paralleling heterogenous enhancement seen in CT.

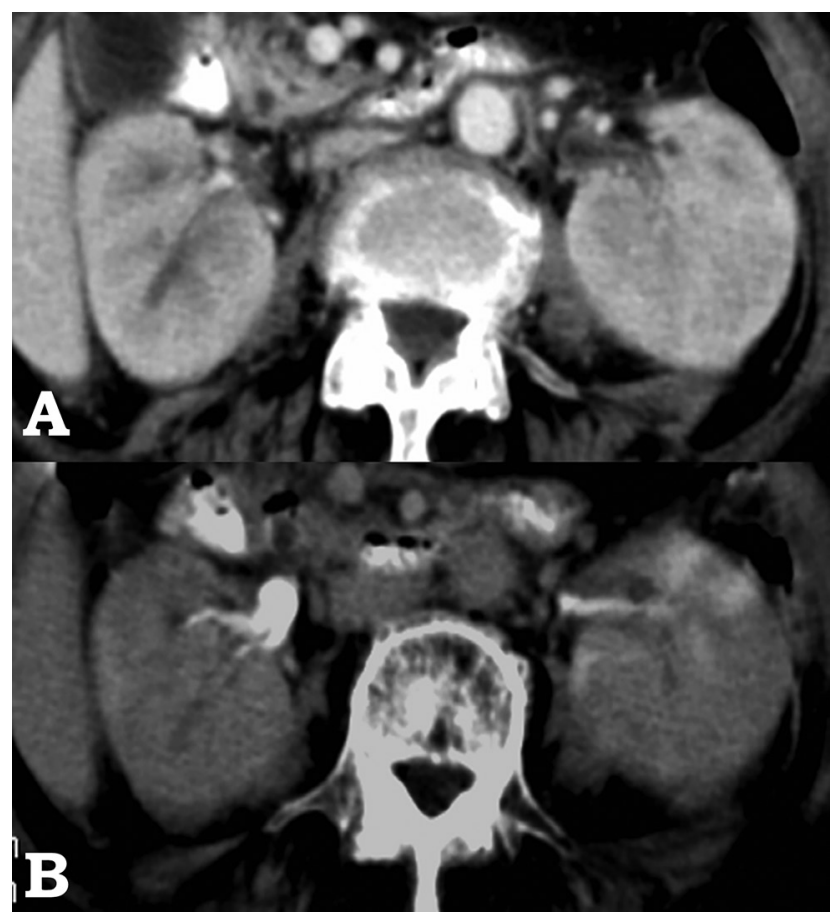

Fig. 5 (A and B) Axial CT sections: Initial nephrographic phase image (A) fails to depict patchy parenchymal enhancement of kidneys that is depicted in 10 minutes excretory phase images (B). Note can be made of perinephric fat stranding around left kidney and urothelial thickening of left renal pelvis.

\section{Renal Microabscesses}

Small microabscesses are seen in almost a third of cases of AP. These may coalesce and form larger abscesses and are well picked up by both CT and DWI ( - Figs. 7 and $\mathbf{8}$ ).

\section{Urothelial Thickening}

Subtle urothelial thickening and increased urothelial enhancement in the walls of the renal pelvis as well as the ureter and along the mucosal layer of the urinary bladder can be well seen on CT and aid the diagnosis of AP especially in cases where renal changes are mild ( $\boldsymbol{\sim}$ Fig. $\mathbf{9}$ ).

\section{Renal Abscess}

Renal abscess is a relatively rare consequence of treated cases of AP. But in poorly treated or inappropriately treated cases and in immunocompromised, especially in 


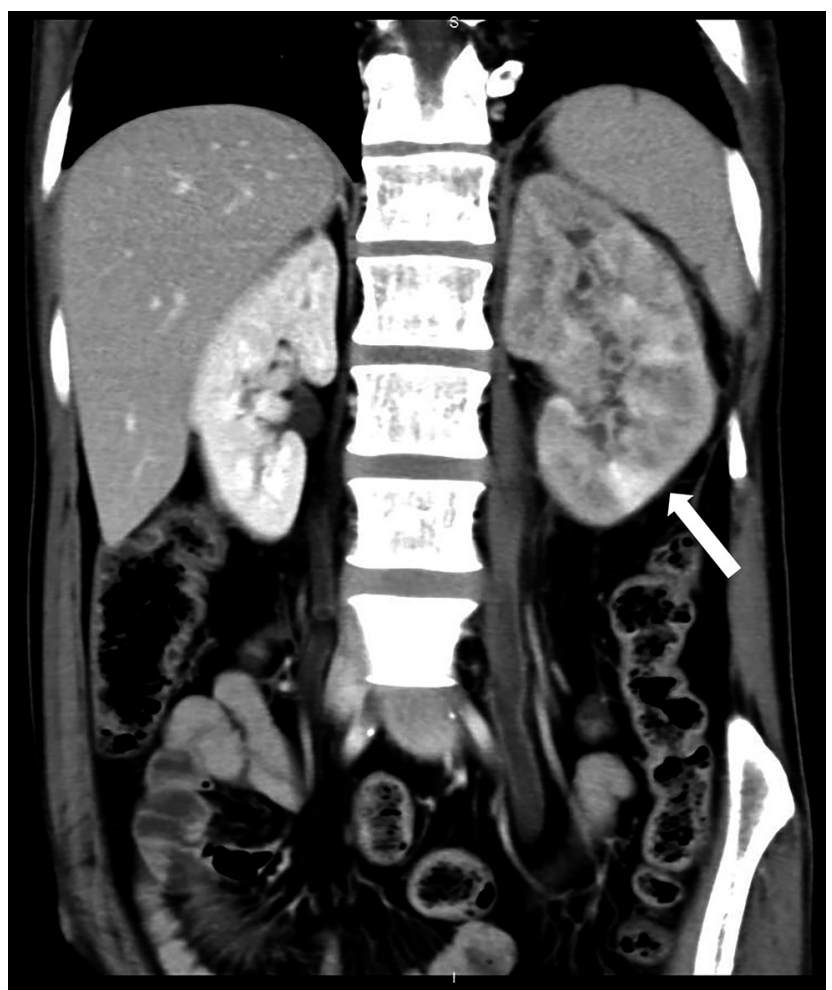

Fig. 6 Coronal axial CT section in the nephrographic phase in a 23-year-old man reveals a significantly swollen left kidney with reduced enhancement (arrow).

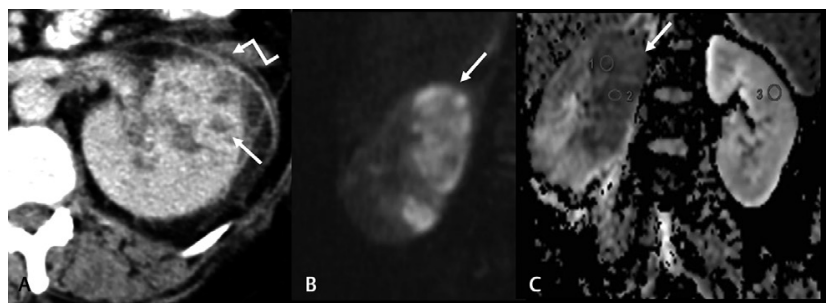

Fig. 7 (A) An axial CT reveals swollen kidney with small microabscesses (straight arrow), perirenal fascial thickening (elbow arrow), and dirty fat around kidney suggesting inflammation. (B) In a different case, microabscesses are (C) showing restricted diffusion in diffusion-weighted magnetic resonance imaging seen as hyperintense areas with corresponding lowered apparent diffusion coefficient (ADC) (arrow) in affected renal parenchyma. ADC value of inflamed parenchyma in right kidney measures $0.93 \times 10^{-3} \mathrm{~mm}^{2} / \mathrm{sec}$ to $0.95 \times 10^{-3} \mathrm{~mm}^{2} / \mathrm{s}$ and in contralateral normal left kidney, it is $2.0 \times 10^{-3} \mathrm{~mm}^{2} / \mathrm{s}$.

diabetics, renal abscess is a relatively frequent unwanted consequence. Hematogenous infections cause multiple abscesses (- Fig. 10). CT is the modality of choice showing intrarenal loculations as well as extrarenal extent. There is excellent depiction of pus by DWI ( - Figs. 7B and C), which can be performed for patients who are unfit for contrast CT or where radiation exposure is a concern like in pregnant women and children. On CT renal parenchyma around the abscess cavity may appear poorly enhanced on the nephrogram phase due to edema. This hypoattenuating subjacent renal parenchyma may enhance slowly in delayed images excluding necrosis ( - Fig. 11A). Multiple abscesses may be seen where septic emboli seed the kidney. Presence of air

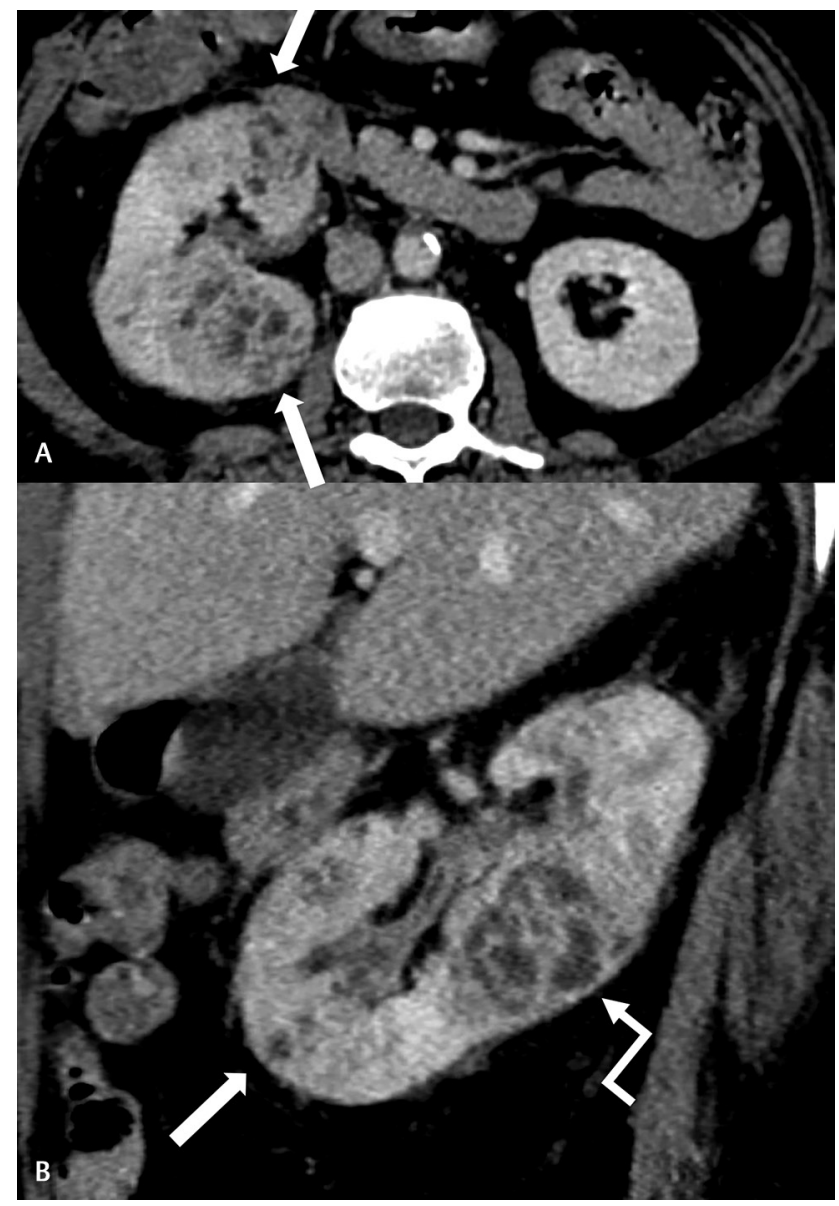

Fig. 8 Panels $\mathbf{A}$ (axial) and $\mathbf{B}$ (sagittal) CT sections in a 68-year-old diabetic woman reveal clusters of tiny cysts representing microabscesses (straight arrows). Coalescing microabscesses evolving into a larger abscess are also seen (elbow arrow)

within the abscesses, collecting system, and the parenchyma is a sign of fulminant infection and called emphysematous pyelonephritis. This will be dealt in the upcoming part 2 of our article ( - Fig. 11B).

\section{Postinflammatory Noninfected Fluid Collections}

Postinflammatory cystic fluid collection may develop in an area of AP during or after antibiotic treatment $(-$ Fig. 12A and B). Renal abscess needs to be differentiated from postinfective sterile fluid collections that are sometimes seen following effective treatment. The principal differentiating point between renal abscess and postinflammatory sterile fluid is enumerated in - Table $\mathbf{1}$.

\section{Extrarenal Extension of Abscess}

Spread of renal infection to perinephric spaces following abscess rupture is not uncommon. Abscess may extend to involve the perinephric spaces, the posterior abdominal wall, or psoas muscle ( - Fig. 13A and B).

\section{Obstructing Pathology}

Presence of obstructing calculus is a risk factor for developing infection. Obstruction of ureter causes increased tissue pressure in kidney that in turn impedes microcirculation and 
that leads to reduced ability to clear infection. It is important to image the urinary tract for any calculi, ureteric stricture, or mass that predisposes to urinary stasis and subsequent pyelonephritis (-Figs. 14-16).

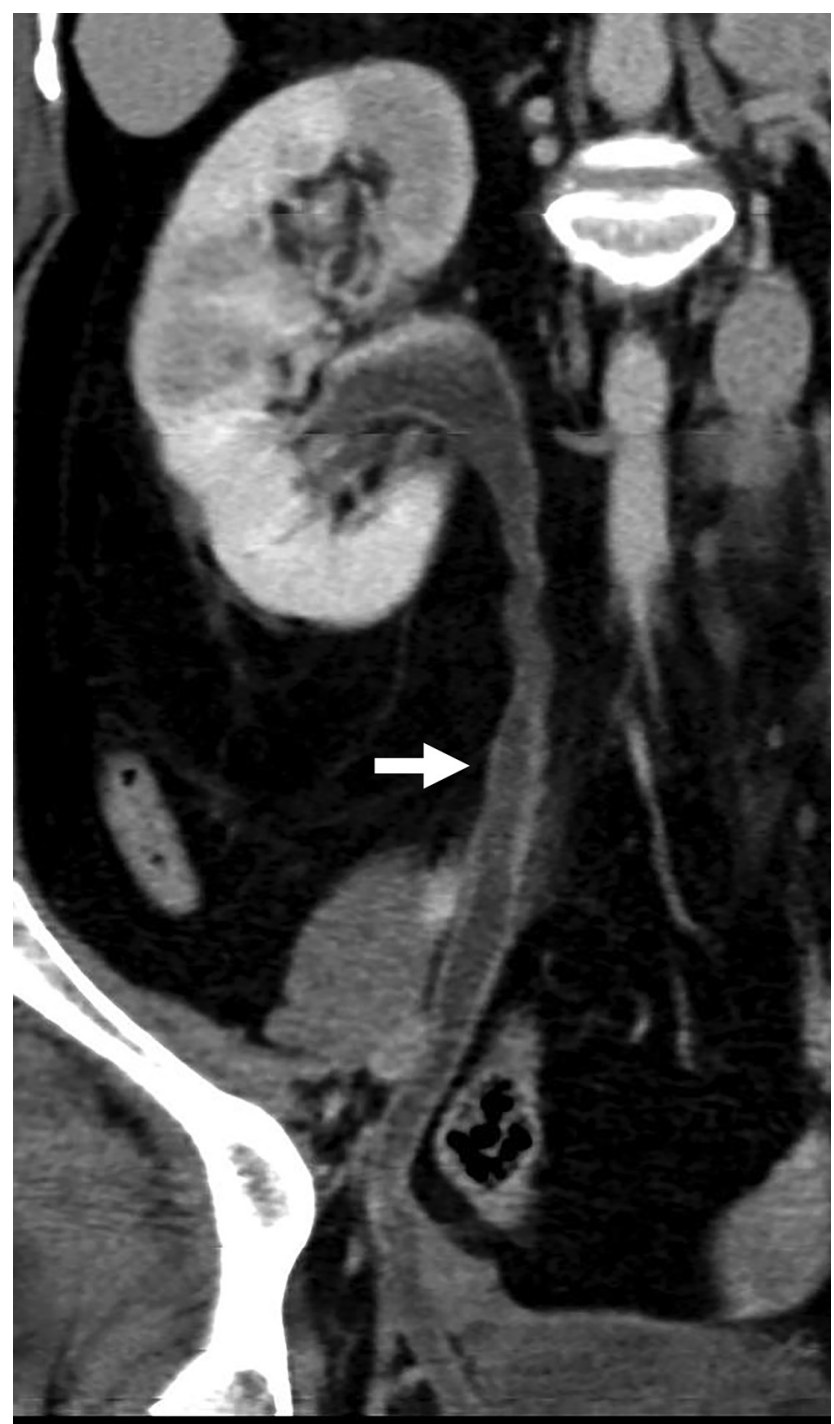

Fig. 9 Curved sagittal CT reconstruction in the nephrographic phase through the right kidney and ureter of a 51 -year-old diabetic woman show mild diffuse urothelial thickening and increased enhancement of the renal pelvis and ureter (arrow).

\section{Pyonephrosis}

Pyonephrosis meaning pus within the collecting system is a situation where obstructed dilated collecting system is laden with pus. USG depiction of debris within a dilated collecting system or documentation of diffusion restriction in MRI helps to establish diagnosis. CT may detect thickened walls of the renal pelvis and ureter, hyperdense urine within the collecting system suggestive of debris, fluid-fluid levels (pus-urine,
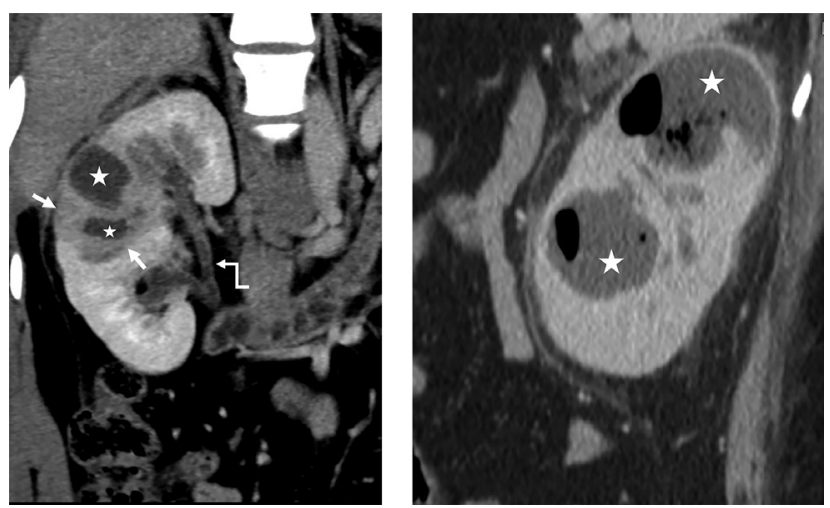

Fig. 11 Coronal nephrographic CT through the right kidney in a 25-year-old woman reveals two abscesses (star) and the perifocal edema around them (straight arrows). Subtle urothelial thickening and enhancement are also seen (elbow arrow)

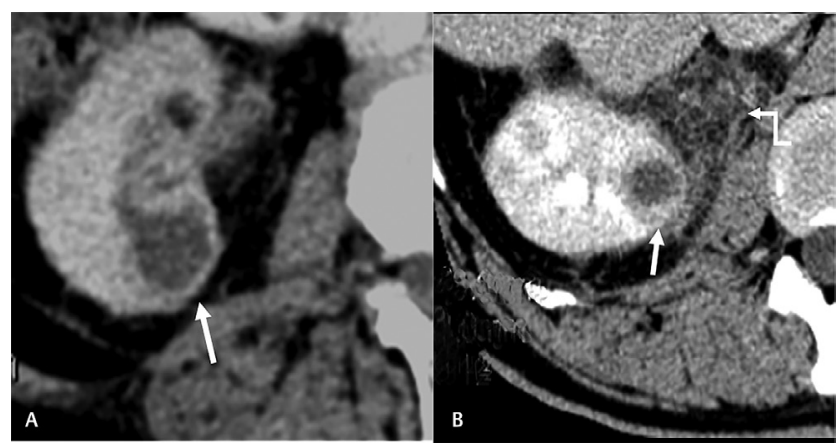

Fig. 12 Axial nephrographic CT sections in a 55-year-old man showing post-infective fluid collection without enhancing wall or hypoperfused surrounding parenchyma (A) and in another 36-year-old woman showing right renal abscess with hypoperfusion of perifocal parenchyma and extensive perinephric fat stranding signifying acute pathology (B).

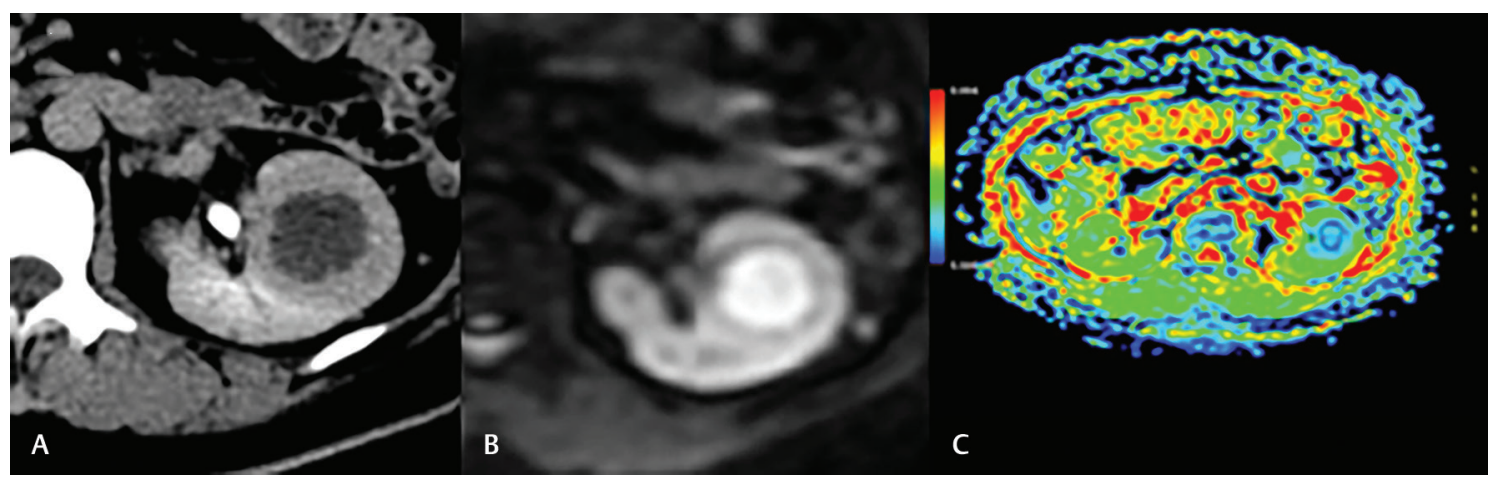

Fig. 10 (A) Axial nephrographic CT section in a 56-year-old diabetic man reveals a thick-walled abscess with surrounding hypoperfused parenchyma. (B) Diffusion-weighted magnetic resonance imaging reveals restricted diffusion and low apparent diffusion coefficient $0.71 \times 10^{-3}$ $\mathrm{mm} /(\mathbf{C})$. 


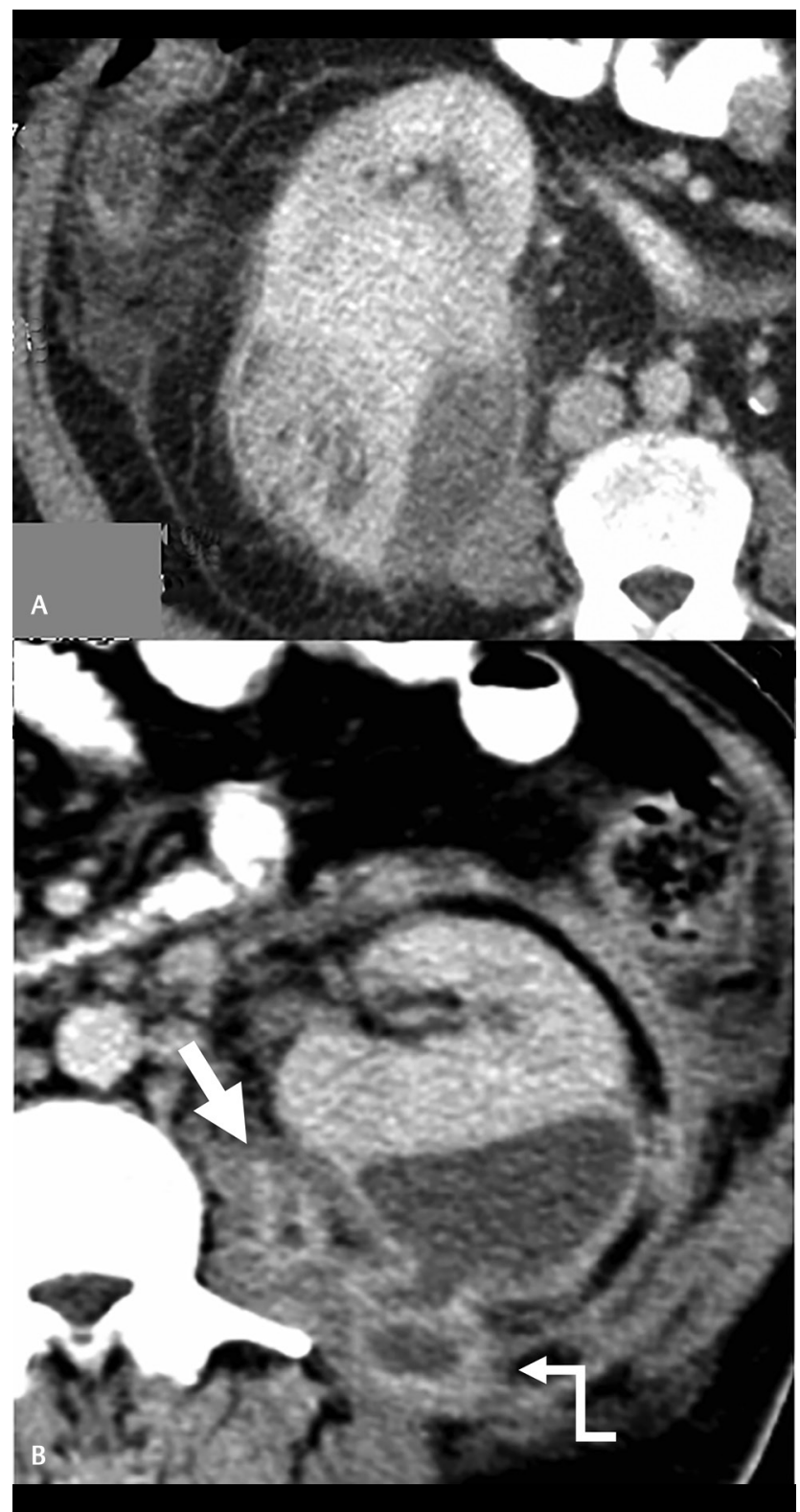

Fig. 13 (A) Axial nephrographic CT in a 64-year-old diabetic man with poor glycemic control shows a large renal abscess with perinephric extension of inflammation. (B) Axial nephrographic CT in another 62-yearold diabetic woman shows a subcapsular rupture of the abscess in the left kidney with extension into posterior abdominal wall (elbow arrow) and left psoas muscle (straight arrow).
Table 1 Differentiating postinflammatory fluid and renal abscess

\begin{tabular}{|l|l|}
\hline Postinflammatory sterile fluid & Renal abscess \\
\hline - $<3 \mathrm{~cm}$ in diameter & - No size criteria \\
- No wall thickening or & - Wall thickening and \\
enhancement & enhancement \\
- Perinephric fat remains clean & - Perinephric fat strand- \\
- Aspiration shows brownish & ing common \\
fluid & - Aspiration reveals pus \\
- USG reveals anechoic fluid & - USG reveals debris \\
without debris & - Restricted diffusion \\
MRI diffusion restriction in & in MRI \\
\hline
\end{tabular}

Abbreviations: MRI, magnetic resonance imaging; USG, ultrasonography.

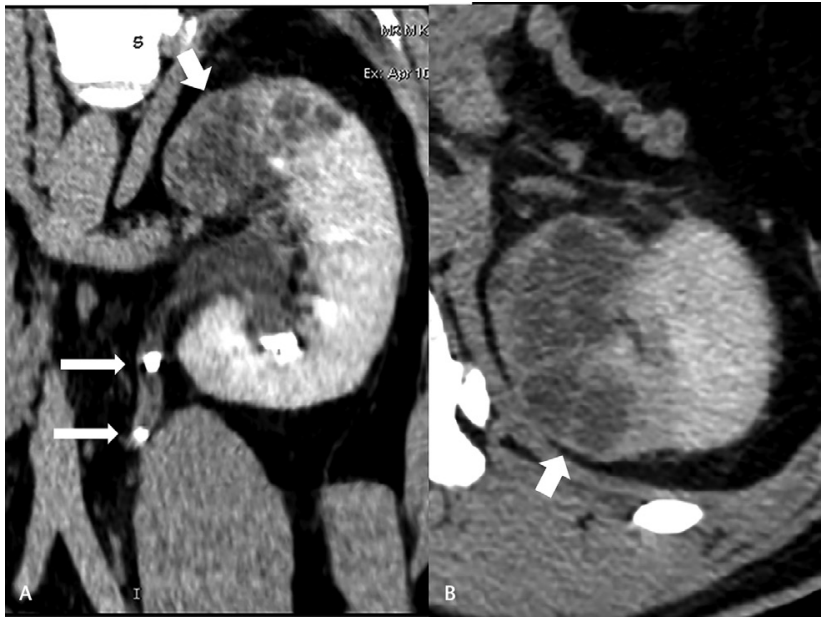

Fig. 14 Coronal (A) and axial (B) nephrographic CT image of left kidney of a 43-year-old man with obstructed renal collecting system by ureteric calculi (long arrows). There is infection in the upper pole with abscess formation (short arrow)

urine-debris, or contrast-debris levels), and gas within the collecting system to contribute to a diagnosis ( - Fig. 17).

\section{Infected Renal Cysts}

Infected renal cysts cannot be reliably differentiated radiologically from an abscess. ${ }^{28,29} \mathrm{An}$ infected cyst may also mimic a renal cyst complicated by intracystic hemorrhage on imaging studies. Percutaneous cyst aspiration and drainage

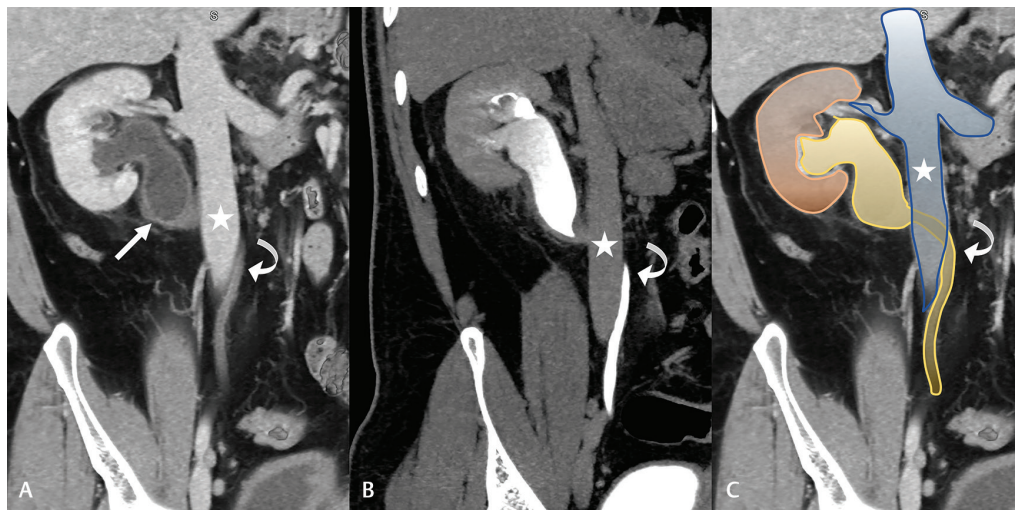

Fig. 15 Coronal oblique CT volume rendered nephrographic phase (A), maximum intensity projection excretory phase (B), and diagrammatic representation (C) through the right kidney and ureter of a 29-year-old man with acute pyelonephritis show a retrocaval ureter (curved arrows) causing hydronephrosis. The inferior vena cava is marked with a star. The straight arrow in panel A shows urothelial thickening of the pelvic wall signifying infection. 


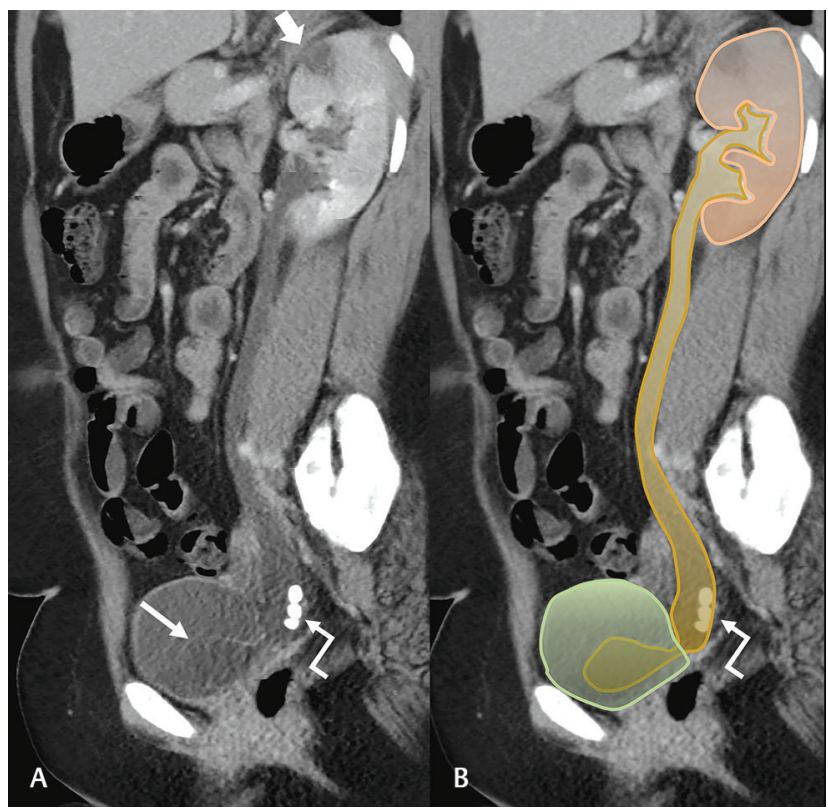

Fig. 16 Sagittal curved CT reconstruction in the nephrographic phase (A) and diagrammatic representation (B) through the left kidney, ureter, and bladder in a 33-year-old lady with acute pyelonephritis and hydronephrosis show a ureterocele (long arrow) as the cause of the obstruction. The upper pole of the kidney reveals a poorly enhancing area suggestive of infection (short stout arrow). Three loose lying nonobstructive calculi are seen in the distal dilated ureter (elbow arrows).

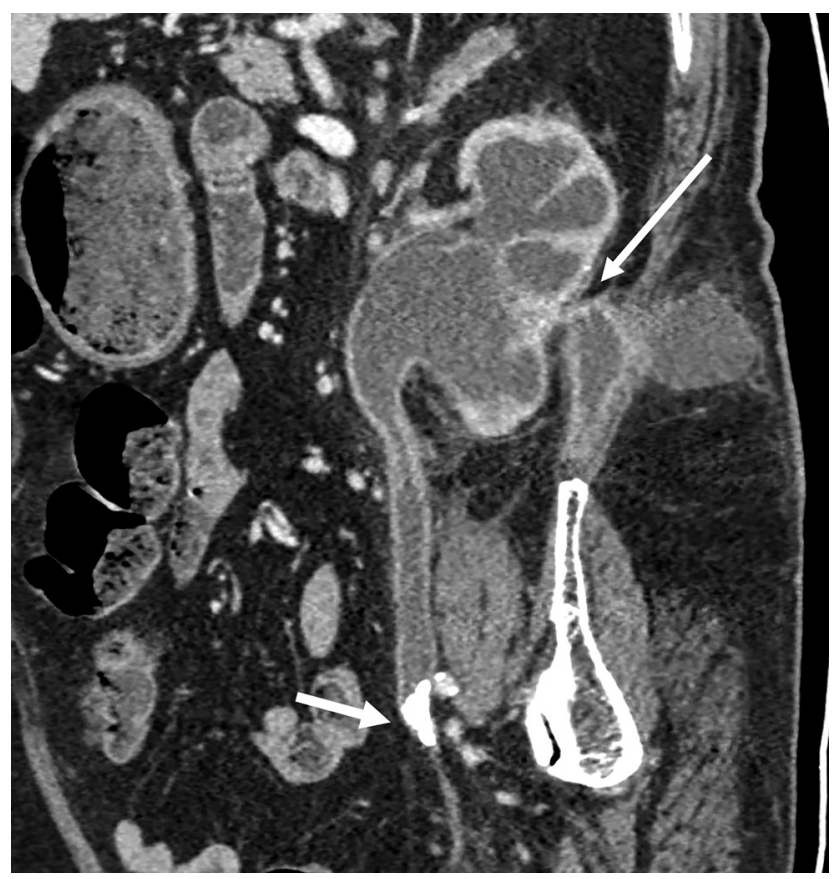

Fig. 17 Oblique coronal CT reconstruction in the nephrographic phase through the left kidney and ureter of a 78-year-old man with chronic left ureteric obstruction by calculi (short arrow). The urine reveals high density ( $25 \mathrm{HU}$ ) suggestive of debris signifying a diagnosis of pyonephrosis. An abscess is seen leading from one of the posterior calyces into the abdominal wall muscles at the back (long arrow).

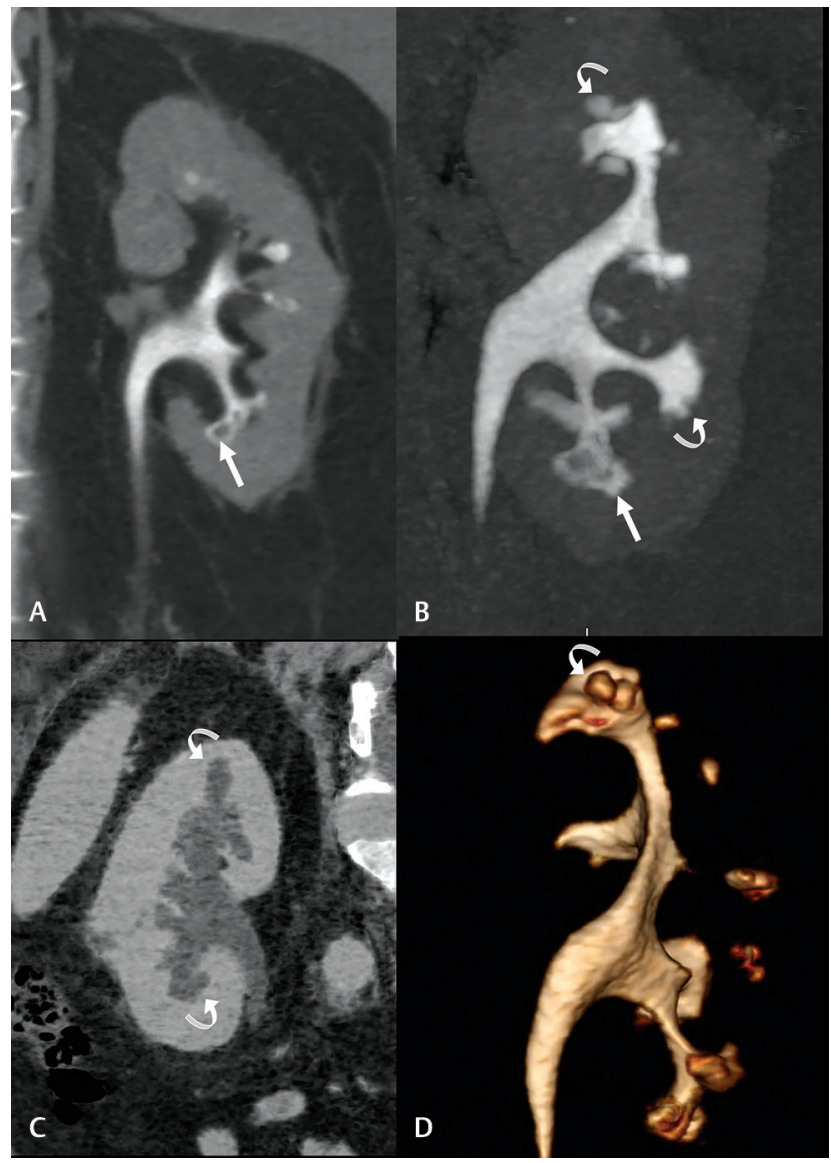

Fig. 18 Sagittal CT maximum intensity (A), volume rendered (B), and colored volume rendered (C) images through the left kidney in the excretory phase of a 50 -year-old diabetic woman with acute pyelonephritis show deformed ballooned calyces with filling defects (straight arrows) along with medullary clefts and deformities of the calyces (curved arrows). A minimum intensity projection through the right kidney in the plain scan (D) of another 68-year-old man with deranged renal functions shows pelvicalyceal mild dilatation along with clubbing and deformity of several calyces (curved arrows).

under CT or US guidance may be indicated for diagnosis and treatment.

\section{Papillary Necrosis}

AP is one of several diseases such as diabetes, analgesic overuse, sickle cell disease, renal vein thrombosis, tuberculosis, and obstructive uropathy that cause renal papillary necrosis. ${ }^{30}$ CT findings include contrast filled clefts in the renal medulla in the excretory phase, nonenhanced lesions surrounded by rings of excreted contrast material representing sloughed off medullae, and medullary calcifications. Sloughed off papillae may also be seen in the renal pelvis or ureter and may cause ureteric obstruction ( - Fig. 18).

An imaging algorithm for patients with AP has been provided in - Fig. 19 


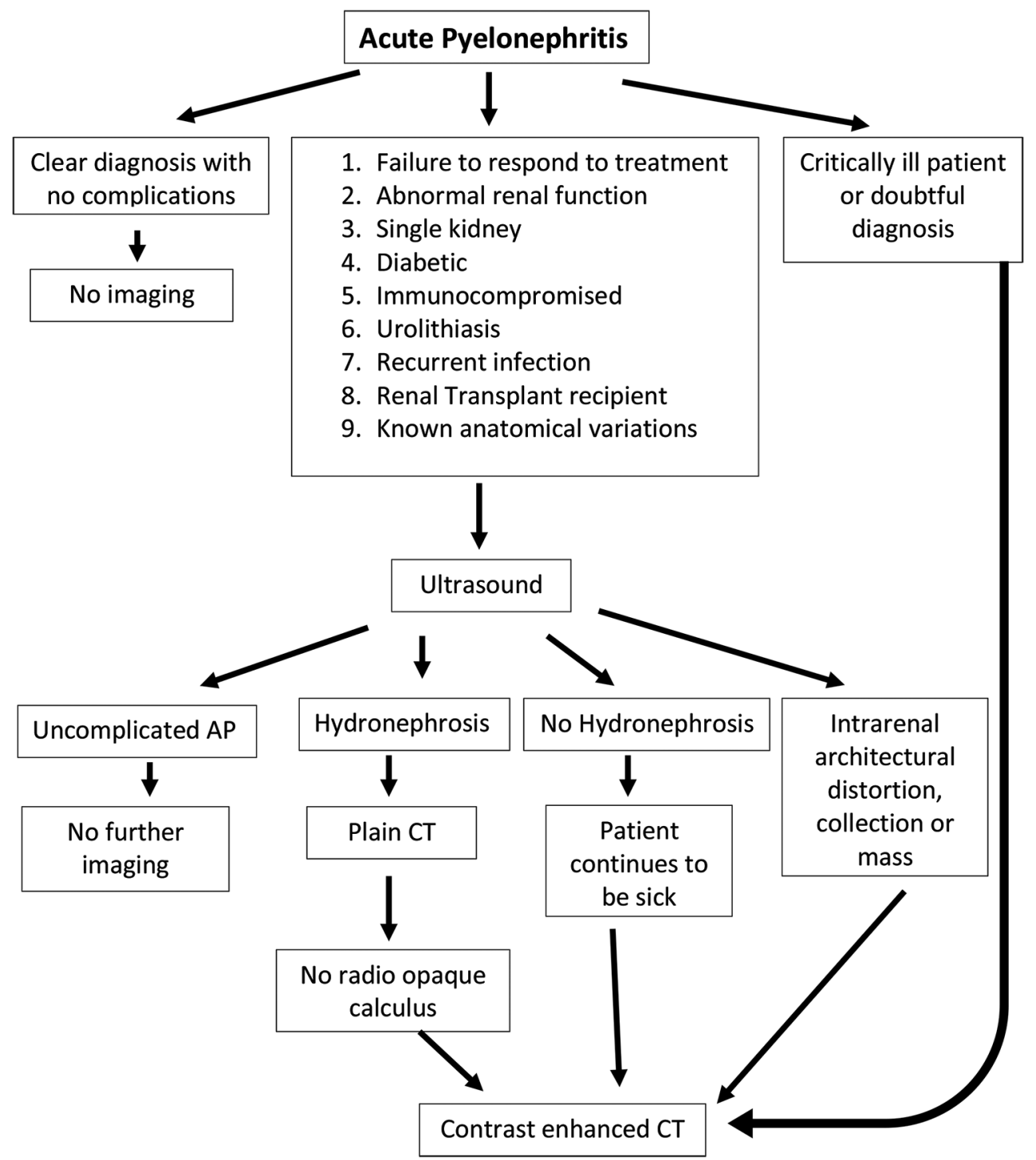

Fig. 19 Acute pyelonephritis imaging approach. AP, acute pyelonephritis; CT, computed tomography.

\section{Conclusion}

Early detection of AP is important to avoid complications especially in vulnerable groups of patients. A USG helps to rule out obstruction in most patients. CT helps in patients who have complicated AP, have not responded to treatment, or are very sick. The sensitivity and specificity of CT for early detection of AP and mapping extra renal extent of disease process are high. MRI is reserved for patients where $\mathrm{CT}$ is contraindicated.

\section{Conflict of Interest}

None declared.

\section{References}

1 Nicolle LE. Complicated pyelonephritis: unresolved issues. Curr Infect Dis Rep 2007;9(6):501-507

2 Kawashima A, Sandler CM, Goldman SM. Imaging in acute renal infection. BJU Int 2000;86(Suppl 1):70-79
3 Davidson AJ, Talner LB. Urographic and angiographic abnormalities in adult-onset acute bacterial nephritis. Radiology 1973;106(2):249-256

4 Lee JK, McClennan BL, Melson GL, Stanley RJ. Acute focal bacterial nephritis: emphasis on gray scale sonography and computed tomography. AJR. Am J Roentgenol 1980;135(1):87-92

5 Rosenfield AT, Glickman MG, Taylor KJ, Crade M, Hodson J. Acute focal bacterial nephritis (acute lobar nephronia). Radiology 1979;132(3):553-561

6 Talner LB, Davidson AJ, Lebowitz RL, Dalla Palma L, Goldman SM. Acute pyelonephritis: can we agree on terminology? Radiology 1994;192(2):297-305

7 Scholes D, Hooton TM, Roberts PL, Gupta K, Stapleton AE, Stamm WE. Risk factors associated with acute pyelonephritis in healthy women. Ann Intern Med 2005;142(1):20-27

8 Hill JB, Sheffield JS, McIntire DD, Wendel GD, Jr. Acute pyelonephritis in pregnancy. Obstet Gynecol 2005;105(1):18-23

9 Ulleryd P. Febrile urinary tract infection in men. Int J Antimicrob Agents 2003;22(Suppl 2):89-93

10 Cattell WR. Urinary tract infection and acute renal failure. In: Raine AE, eds. Advanced Renal Medicine. Oxford: Oxford University Press; 1992:302-313 
11 Fulop T. Acute Pyelonephritis Workup. Aug 22, 2012. Available at: http://emedicine.medscape.com/article/245559workup\#aw2aab6b5b3. Accessed February 2015

12 June $\mathrm{CH}$, Browning MD, Smith LP, et al. Ultrasonography and computed tomography in severe urinary tract infection. Arch Intern Med 1985;145(5):841-845

13 Soulen MC, Fishman EK, Goldman SM, Gatewood OM. Bacterial renal infection: role of CT. Radiology 1989;171(3):703-707

14 Kawashima A, Sandler CM, Ernst RD, Goldman SM, Raval B, Fishman EK. Renal inflammatory disease: the current role of CT. Crit Rev Diagn Imaging 1997;38(5):369-415

15 Kawashima A, Sandler CM, Goldman SM. Current roles and controversies in the imaging evaluation of acute renal infection. World J Urol 1998;16(1):9-17

16 Hoepelman AI, Meiland R, Geerlings SE. Pathogenesis and management of bacterial urinary tract infections in adult patients with diabetes mellitus. Int $\mathrm{J}$ Antimicrob Agents 2003;22(Suppl 2):35-43

17 Mitra S, Acharya H, Dua J, et al. Role of emergency ultrasound screening in the management of acute pyelonephritis in emergency department: A large observational study from a tertiary care center of South India. J Med Soc 2017;31:43-47

18 American College of Radiology. ACR Appropriateness criteria. Acute Pyelonephritis. Available at: https://acsearch.acr.org/ docs/69489/Narrative/

19 Demertzis J, Menias CO. State of the art: imaging of renal infections. Emerg Radiol 2007;14(1):13-22

20 Craig WD, Wagner BJ, Travis MD. Pyelonephritis: radiologic-pathologic review. Radiographics 2008;28(1):255-277, quiz 327-328

21 Vivier PH, Sallem A, Beurdeley M, et al. MRI and suspected acute pyelonephritis in children: comparison of diffusion-weighted imaging with gadolinium-enhanced T1-weighted imaging. Eur Radiol 2014;24(1):19-25

22 Rathod SB, Kumbhar SS, Nanivadekar A, Aman K. Role of diffusion-weighted MRI in acute pyelonephritis: a prospective study. Acta Radiol 2015;56(2):244-249

23 De Pascale A, Piccoli GB, Priola SM, et al. Diffusion-weighted magnetic resonance imaging: new perspectives in the diagnostic pathway of non-complicated acute pyelonephritis. Eur Radiol 2013;23(11):3077-3086

24 Chan JH, Tsui EY, Luk SH, et al. MR diffusion-weighted imaging of kidney: differentiation between hydronephrosis and pyonephrosis. Clin Imaging 2001;25(2):110-113

25 Thoeny HC, De Keyzer F, Oyen RH, Peeters RR. Diffusion-weighted MR imaging of kidneys in healthy volunteers and patients with parenchymal diseases: initial experience. Radiology 2005;235(3):911-917

26 Stunell H, Buckley O, Feeney J, Geoghegan T, Browne RF, Torreggiani WC. Imaging of acute pyelonephritis in the adult. Eur Radiol 2007;17(7):1820-1828

27 Taniguchi LS, Torres US, Souza SM, Torres LR, D'Ippolito G. Are the unenhanced and excretory CT phases necessary for the evaluation of acute pyelonephritis? Acta Radiol 2017;58(5):634-640

28 Feldberg MA, Mali WP. An infected renal cyst. Urol Radiol 1980;2(1):47-49

29 Frishman E, Orron DE, Heiman Z, Kessler A, Kaver I, Graif M. Infected renal cysts: sonographic diagnosis and management. J Ultrasound Med 1994;13(1):7-10

30 Jung DC, Kim SH, Jung SI, Hwang SI, Kim SH. Renal papillary necrosis: review and comparison of findings at multi-detector row CT and intravenous urography. Radiographics 2006;26(6):1827-1836 